\title{
Some remarks on the Plotkin bound
}

\author{
Jörn Quistorff \\ Speckenreye 48 \\ 22119 Hamburg, Germany \\ joern.quistorff@hamburg.de
}

Submitted: Nov 24, 2001; Accepted: Jun 17, 2003; Published: Jun 27, 2003

MR Subject Classifications: 94B65

\begin{abstract}
In coding theory, Plotkin's upper bound on the maximal cadinality of a code with minimum distance at least $d$ is well known. He presented it for binary codes where Hamming and Lee metric coincide. After a brief discussion of the generalization to $q$-ary codes preserved with the Hamming metric, the application of the Plotkin bound to q-ary codes preserved with the Lee metric due to Wyner and Graham is improved.
\end{abstract}

\section{Introduction}

Let $K$ be a set of cardinality $q \in \mathbf{N}$ and $d^{K}: K \times K \rightarrow \mathbf{R}$ be a metric. Consider $R:=K^{n}$ with $n \in \mathbf{N}$ and $d^{R}\left(\left(v_{1}, \ldots, v_{n}\right),\left(w_{1}, \ldots, w_{n}\right)\right):=\sum_{i=1}^{n} d^{K}\left(v_{i}, w_{i}\right)$. Then $\left(K, d^{K}\right)$ and $\left(R, d^{R}\right)$ are finite metric spaces.

A subset $C \subseteq R$ is called a (block) code of length $n$. If $|C| \geq 2$ then its minimum distance is defined by $d(C):=\min \left\{d^{R}(v, w) \in \mathbf{R}^{+} \mid v, w \in C\right.$ and $\left.v \neq w\right\}$. The observation of the metric properties of $\left(R, d^{R}\right)$ and of its subsets is an essential part of coding theory. The value $u\left(R, d^{R}, d\right)$ (or briefly $u(d)$ ), defined as the maximal cardinality of a code $C \subseteq R$ with minimum distance $d(C) \geq d$, is frequently considered.

The determination of $u(d)$ is a fundamental and often unsolved problem but some lower and upper bounds are well known. This paper deals with the following condition on the parameters of a code which gives Plotkin's upper bound on $u(d)$. Similar formulations are given by Berlekamp [1] and Răduică [8].

Let $d>0$ and $u \in \mathbf{N} \backslash\{1\}$. Put $J:=\{0, \ldots, u-1\}$. If $u(d) \geq u$ then

$$
d\left(\begin{array}{l}
u \\
2
\end{array}\right) \leq n \max \left\{\sum_{\{j, k\} \subseteq J} d^{K}\left(v_{1}^{(j)}, v_{1}^{(k)}\right) \mid\left(v_{1}^{(0)}, \ldots, v_{1}^{(u-1)}\right) \in K^{u}\right\}=: n P_{\left(K, d^{K}\right)}(u) .
$$

This condition is easy to prove by estimating $\sum_{\{v, w\} \subseteq C} d^{R}(v, w)$. 
If instead of $P_{\left(K, d^{K}\right)}(u)$ an upper bound $Q_{\left(K, d^{K}\right)}(u)$ is known then inequality (1) can be replaced by

$$
d\left(\begin{array}{l}
u \\
2
\end{array}\right) \leq n Q_{\left(K, d^{K}\right)}(u) .
$$

The most common finite metric spaces in coding theory are the ( $n$-dimensional $q$-ary) Hamming spaces $\left(R, d_{H}\right)$. Here, the Hamming metric can be introduced by

$$
d_{H}\left(\left(v_{1}, \ldots, v_{n}\right),\left(w_{1}, \ldots, w_{n}\right)\right)=\sum_{i=1}^{n} d_{H}\left(v_{i}, w_{i}\right)
$$

and

$$
d_{H}\left(v_{i}, w_{i}\right)= \begin{cases}0 & \text { if } v_{i}=w_{i} \\ 1 & \text { if } v_{i} \neq w_{i}\end{cases}
$$

Furthermore, $A_{q}(n, d)$ is usually used instead of $u\left(R, d_{H}, d\right)$.

Other common finite metric spaces in coding theory consider $R=K^{n}$ with $K=\mathbf{Z} / q \mathbf{Z}$ together with the Lee metric $d_{L}$ which can be introduced by

$$
d_{L}\left(\left(v_{1}, \ldots, v_{n}\right),\left(w_{1}, \ldots, w_{n}\right)\right)=\sum_{i=1}^{n} d_{L}\left(v_{i}, w_{i}\right)
$$

and

$$
d_{L}\left(v_{i}, w_{i}\right)=\min \left\{\left|v_{i}-w_{i}\right|, q-\left|v_{i}-w_{i}\right|\right\} .
$$

Whenever, like on the right-hand side of equation (3), an order $\leq$ is used in $\mathbf{Z} / p \mathbf{Z}$, their elements have to be represented by elements of $\{0, \ldots, p-1\} \subseteq \mathbf{Z}$. The spaces $\left(R, d_{L}\right)$ should be called Lee spaces.

In case of $q \leq 3$, the metrics $d_{H}$ and $d_{L}$ are identical. Lee [3] noticed that also the case $\left((\mathbf{Z} / 4 \mathbf{Z})^{n}, d_{L}\right)$ can be reduced to $\left((\mathbf{Z} / 2 \mathbf{Z})^{2 n}, d_{H}\right)$, using the transformation $0 \mapsto(0,0)$, $1 \mapsto(0,1), 2 \mapsto(1,1), 3 \mapsto(1,0)$. The pathological case $q=1$ is usually omitted.

After a brief discussion of the Plotkin bound in Hamming spaces, the paper considers this bound in Lee spaces.

\section{Hamming Spaces}

Plotkin [6] introduced his bound in case of $q=2$ where Hamming and Lee metric coincide. In terms of condition (1), he used $P_{2}^{H}(u):=P_{\left(\{0,1\}, d_{H}\right)}(u)=\left\lfloor\frac{u+1}{2}\right\rfloor\left(u-\left\lfloor\frac{u+1}{2}\right\rfloor\right)$ and proved the existence of an $m \in \mathbf{N}$ with

$$
A_{2}(n, d) \leq 2 m \leq \frac{2 d}{2 d-n}
$$

if $2 d>n$. MacWilliams/Sloane [5] mentioned in this case the equivalent bound

$$
A_{2}(n, d) \leq 2\left\lfloor\frac{d}{2 d-n}\right\rfloor
$$


Berlekamp [1] considered the generalization to $q$-ary Hamming spaces. In terms of $P_{q}^{H}:=P_{\left(\mathbf{Z} / q \mathbf{Z}, d_{H}\right)}$ and $Q_{q}^{H}$, he showed $P_{q}^{H}(u) \leq Q_{q}^{H}(u)=\frac{u^{2}(q-1)}{2 q}$. This result yields the bound

$$
A_{q}(n, d) \leq \frac{d q}{d q-n(q-1)} \quad \text { if } \quad d q>n(q-1) .
$$

Quistorff [7] determined

$$
P_{q}^{H}(u)=\left(\begin{array}{l}
u \\
2
\end{array}\right)-b\left(\begin{array}{c}
a+1 \\
2
\end{array}\right)-(q-b)\left(\begin{array}{l}
a \\
2
\end{array}\right)
$$

if $u=a q+b$ with $a, b \in \mathbf{N}_{0}$ and $b<q$. An equivalent statement can be found in Bogdanova et al. [2]. The results (1) and (6) imply e.g. the tight upper bound $A_{3}(9,7) \leq 6$. Vaessens/Aarts/Van Lint [9] formerly mentioned this and similar examples for $q=3$ as an implication of Plotkin [6] and also solved the case $a=b=1$ in (6) with arbitrary $q \in \mathbf{N} \backslash\{1\}$. Mackenzie/Seberry's [4] bound on $A_{3}(n, d)$ with $3 d>2 n$ is incorrect. The adequate use of their method leads to

$$
A_{3}(n, d) \leq \max \left\{3\left\lfloor\frac{d}{3 d-2 n}\right\rfloor, 3\left\lfloor\frac{d}{3 d-2 n}-\frac{2}{3}\right\rfloor+1\right\} \quad \text { if } \quad 3 d>2 n
$$

which is equivalent to the application of (6).

\section{$3 \quad$ Lee Spaces}

Put $P_{q}^{L}(u):=P_{\left(\mathbf{Z} / q \mathbf{Z}, d_{L}\right)}(u)$. Wyner/Graham $[10]$ proved

$$
P_{q}^{L}(u) \leq Q_{q}^{L}(u):= \begin{cases}\frac{u^{2}\left(q^{2}-1\right)}{8 q} & \text { if } q \text { is odd } \\ \frac{u^{2}}{8} q & \text { if } q \text { is even }\end{cases}
$$

as an application of the Plotkin bound in Lee spaces, cf. also Berlekamp [1]. The stronger inequality

$$
P_{q}^{L}(u) \leq\left\lfloor Q_{q}^{L}(u)\right\rfloor
$$

follows by definition. In order to improve formula (7), some preparation is necessary.

Lemma 1 Let $q, u \in \mathbf{N} \backslash\{1\}$ and $m \in\{1, \ldots, u-1\}$. Let $J:=\mathbf{Z} / u \mathbf{Z}$ and $v^{(j)} \in \mathbf{Z} / q \mathbf{Z}$ with $j \in J$ and $v^{(j)} \leq v^{(k)}$ for $j<k$. Then

$$
\sum_{j \in J} d_{L}\left(v^{(j)}, v^{(j+m)}\right) \leq m q
$$

and equality holds in estimation (8) iff

$$
d_{L}\left(v^{(j)}, v^{(j+m)}\right)= \begin{cases}v^{(j+m)}-v^{(j)} & \text { if } j<u-m \\ q+v^{(j+m)}-v^{(j)} & \text { if } j \geq u-m\end{cases}
$$

is valid. 
Proof:

$$
\sum_{j \in J} d_{L}\left(v^{(j)}, v^{(j+1)}\right) \leq q+v^{(0)}-v^{(u-1)}+\sum_{j \in J \backslash\{u-1\}} v^{(j+1)}-v^{(j)}=q
$$

and hence

$$
\begin{aligned}
\sum_{j \in J} d_{L}\left(v^{(j)}, v^{(j+m)}\right) & \leq \sum_{j \in J} \sum_{l=0}^{m-1} d_{L}\left(v^{(j+l)}, v^{(j+l+1)}\right) \\
& \leq \sum_{l=0}^{m-1} \sum_{j \in J} d_{L}\left(v^{(j)}, v^{(j+1)}\right) \\
& \leq m q .
\end{aligned}
$$

All estimates turn out to be equalities iff condition (9) is valid.

Put

$$
N_{q}^{L}(u):= \begin{cases}\frac{u^{2}-1}{8} q & \text { if } u \text { is odd } \\ \frac{u(u-2)}{8} q+\frac{u}{2}\left\lfloor\frac{q}{2}\right\rfloor & \text { if } u \text { is even }\end{cases}
$$

with $u \in \mathbf{N} \backslash\{1\}$. Clearly, $\frac{u^{2}-1}{8} \in \mathbf{N}$ if $u$ is odd and $\frac{u(u-2)}{8} \in \mathbf{N}_{0}$ if $u$ is even.

Theorem 2 Let $q, u \in \mathbf{N} \backslash\{1\}$. Then $P_{q}^{L}(u) \leq N_{q}^{L}(u)$ holds true.

Proof: Let $v^{(j)} \in \mathbf{Z} / q \mathbf{Z}$ with $j \in J:=\mathbf{Z} / u \mathbf{Z}$. Without loss of generality, let $v^{(j)} \leq v^{(k)}$ for $j<k$.

(i) Let $u$ be odd. Then

$$
\begin{aligned}
\sum_{\{j, k\} \subseteq J} d_{L}\left(v^{(j)}, v^{(k)}\right) & =\sum_{m=1}^{\frac{u-1}{2}} \sum_{j \in J} d_{L}\left(v^{(j)}, v^{(j+m)}\right) \\
& \leq \sum_{m=1}^{\frac{u-1}{2}} m q=N_{q}^{L}(u)
\end{aligned}
$$

follows by Lemma 1 .

(ii) Let $u$ be even. Then

$$
\begin{aligned}
\sum_{\{j, k\} \subseteq J} d_{L}\left(v^{(j)}, v^{(k)}\right) & =\sum_{m=1}^{\frac{u}{2}-1} \sum_{j \in J} d_{L}\left(v^{(j)}, v^{(j+m)}\right)+\sum_{j \in J ; j<\frac{u}{2}} d_{L}\left(v^{(j)}, v^{\left(j+\frac{u}{2}\right)}\right) \\
& \leq \sum_{m=1}^{\frac{u}{2}-1} m q+\frac{u}{2}\left\lfloor\frac{q}{2}\right\rfloor=N_{q}^{L}(u)
\end{aligned}
$$

follows by Lemma 1 . 
Hence, in both cases $P_{q}^{L}(u) \leq N_{q}^{L}(u)$ is valid.

Theorem 2 improves formula (7) in many cases. E.g. $N_{8}^{L}(3)=8<9=\left\lfloor Q_{8}^{L}(3)\right\rfloor$ and $N_{9}^{L}(6)=39<40=\left\lfloor Q_{9}^{L}(6)\right\rfloor$ hold true.

The following statements will prove coincidence between $P_{q}^{L}(u)$ and $N_{q}^{L}(u)$ if $q$ is odd or $u$ is small, relative to $q$. Put $f(u):=1$ if $u$ is odd and $f(u):=2$ if $u$ is even.

Lemma 3 Let $q, u \in \mathbf{N} \backslash\{1\}$. Let $q$ be even or $f(u) q \geq u-1$. Let $\left\lfloor\frac{j q}{u}\right\rfloor,\left\lfloor\frac{k q}{u}\right\rfloor \in \mathbf{Z} / q \mathbf{Z}$ with $j, k:=j+m \in \mathbf{Z} / u \mathbf{Z}$ and $1 \leq m \leq\left\lfloor\frac{u-1}{2}\right\rfloor$ as well as $0 \leq j, k<u$. Put

$$
\left\lfloor\widetilde{\frac{k q}{u}}\right\rfloor:= \begin{cases}\left\lfloor\frac{k q}{u}\right\rfloor & \text { if } j<u-m \\ q+\left\lfloor\frac{k q}{u}\right\rfloor & \text { if } j \geq u-m .\end{cases}
$$

Then $d_{L}\left(\left\lfloor\frac{j q}{u}\right\rfloor,\left\lfloor\frac{k q}{u}\right\rfloor\right)=\left\lceil\frac{k q}{u}\right\rfloor-\left\lfloor\frac{j q}{u}\right\rfloor \leq\left\lfloor\frac{q}{2}\right\rfloor$ is valid.

Proof: It holds true that $\widetilde{\left.\frac{k q}{u}\right\rfloor} \leq \frac{\left(j+\left\lfloor\frac{u-1}{2}\right\rfloor\right) q}{u}$ and $\left\lfloor\frac{j q}{u}\right\rfloor \geq \frac{j q-(u-1)}{u}$.

(i) Let $u$ be odd. Then $\left.\llbracket \frac{k q}{u}\right\rfloor-\left\lfloor\frac{j q}{u}\right\rfloor \leq\left\lfloor\frac{\frac{u-1}{2} q+(u-1)}{u}\right\rfloor=\left\lfloor\left(\frac{q}{2}+1\right)\left(1-\frac{1}{u}\right)\right\rfloor$. If $q$ is even then $\left\lceil\frac{k q}{u}\right\rfloor-\left\lfloor\frac{j q}{u}\right\rfloor \leq\left\lfloor\frac{q}{2}\right\rfloor$. If $q \geq u-1$ then $\left\lceil\frac{k q}{u}\right\rfloor-\left\lfloor\frac{j q}{u}\right\rfloor \leq\left\lfloor\left(\frac{q}{2}+1\right) \frac{q}{q+1}\right\rfloor=\left\lfloor\frac{q+1}{2}-\frac{1}{2(q+1)}\right\rfloor \leq\left\lfloor\frac{q}{2}\right\rfloor$.

(ii) Let $u$ be even. Then $\left\lceil\frac{k q}{u}\right\rfloor-\left\lfloor\frac{j q}{u}\right\rfloor \leq\left\lfloor\frac{\left(\frac{u}{2}-1\right) q+(u-1)}{u}\right\rfloor=\left\lfloor\left(\frac{q}{2}+1\right)-\frac{q+1}{u}\right\rfloor$. If $q$ is even then $\left\lceil\frac{k q}{u}\right\rfloor-\left\lfloor\frac{j q}{u}\right\rfloor \leq\left\lfloor\frac{q}{2}\right\rfloor$. If $2 q \geq u-1$ then $\left\lceil\frac{k q}{u}\right\rfloor-\left\lfloor\frac{j q}{u}\right\rfloor \leq\left\lfloor\frac{q+1}{2}-\frac{2(q+1)-u}{2 u}\right\rfloor \leq\left\lfloor\frac{q}{2}\right\rfloor$.

Hence, $d_{L}\left(\left\lfloor\frac{j q}{u}\right\rfloor,\left\lfloor\frac{k q}{u}\right\rfloor\right)=\left\lfloor\widetilde{\frac{k q}{u}}\right\rfloor-\left\lfloor\frac{j q}{u}\right\rfloor$.

In case of $q=3, u=5, j=3, m=2$, Lemma 3 can not be applied. Here, $k=0$, $\left\lfloor\frac{j q}{u}\right\rfloor=1,\left\lfloor\frac{k q}{u}\right\rfloor=0,\left\lfloor\frac{k q}{u}\right\rfloor=3,\left\lfloor\frac{k q}{u}\right\rfloor-\left\lfloor\frac{j q}{u}\right\rfloor=2>1=\left\lfloor\frac{q}{2}\right\rfloor$ and $d_{L}\left(\left\lfloor\frac{j q}{u}\right\rfloor,\left\lfloor\frac{k q}{u}\right\rfloor\right)=1$. A similar example is $q=3, u=8, j=5, m=3$.

Lemma 4 Let $q, u \in \mathbf{N} \backslash\{1\}$ and $u$ be even. Let $\left\lfloor\frac{j q}{u}\right\rfloor,\left\lfloor\frac{k q}{u}\right\rfloor \in \mathbf{Z} / q \mathbf{Z}$ with $j, k:=j+\frac{u}{2} \in$ $\mathbf{Z} / u \mathbf{Z}$ and $0 \leq j<\frac{u}{2} \leq k<u$. Then $d_{L}\left(\left\lfloor\frac{j q}{u}\right\rfloor,\left\lfloor\frac{k q}{u}\right\rfloor\right)=\left\lfloor\frac{q}{2}\right\rfloor$ is valid.

Proof: It holds true that $\frac{\left(j+\frac{u}{2}\right) q-(u-1)}{u} \leq\left\lfloor\frac{k q}{u}\right\rfloor \leq \frac{\left(j+\frac{u}{2}\right) q}{u}$ and $\frac{j q-(u-1)}{u} \leq\left\lfloor\frac{j q}{u}\right\rfloor \leq \frac{j q}{u}$. Hence, $\left\lfloor\frac{k q}{u}\right\rfloor-\left\lfloor\frac{j q}{u}\right\rfloor \leq\left\lfloor\frac{q}{2}+\frac{u-1}{u}\right\rfloor \leq\left\lfloor\frac{q+1}{2}\right\rfloor$ and $q-\left\lfloor\frac{k q}{u}\right\rfloor+\left\lfloor\frac{j q}{u}\right\rfloor \leq\left\lfloor\frac{q}{2}+\frac{u-1}{u}\right\rfloor \leq\left\lfloor\frac{q+1}{2}\right\rfloor$. This yields $d_{L}\left(\left\lfloor\frac{j q}{u}\right\rfloor,\left\lfloor\frac{k q}{u}\right\rfloor\right)=\left\lfloor\frac{q}{2}\right\rfloor$.

Theorem 5 Let $q, u \in \mathbf{N} \backslash\{1\}$. Let $q$ be even or $f(u) q \geq u-1$. Then $P_{q}^{L}(u)=N_{q}^{L}(u)$.

Proof: Put $v^{(j)}:=\left\lfloor\frac{j q}{u}\right\rfloor$ for $j \in J:=\mathbf{Z} / u \mathbf{Z}$ with $0 \leq j<u$. 
(i) Let $u$ be odd. Then

$$
\begin{aligned}
P_{q}^{L}(u) \geq \sum_{\{j, k\} \subseteq J} d_{L}\left(v^{(j)}, v^{(k)}\right) & =\sum_{m=1}^{\frac{u-1}{2}} \sum_{j \in J} d_{L}\left(v^{(j)}, v^{(j+m)}\right) \\
& =\sum_{m=1}^{\frac{u-1}{2}} m q \\
& =N_{q}^{L}(u)
\end{aligned}
$$

by Lemma 1 and 3 .

(ii) Let $u$ be even. Then

$$
\begin{aligned}
P_{q}^{L}(u) \geq \sum_{\{j, k\} \subseteq J} d_{L}\left(v^{(j)}, v^{(k)}\right) & \\
& =\sum_{m=1}^{\frac{u}{2}-1} \sum_{j \in J} d_{L}\left(v^{(j)}, v^{(j+m)}\right)+\sum_{j \in J ; j<\frac{u}{2}} d_{L}\left(v^{(j)}, v^{\left(j+\frac{u}{2}\right)}\right) \\
& =N_{q}^{L}(u)
\end{aligned}
$$

by Lemma 1, 3 and 4 .

Theorem 2 completes the proof.

If $u$ is considerable greater than $q$, the Plotkin bound is usually weak and other well known upper bounds, e.g. the Hamming bound, give stronger results. Hence, it seems not to be fatal that $P_{q}^{L}(u)$ is not determined in all these cases. The final theorem gives at least a lower bound on $P_{q}^{L}(u)$. According to Theorem 5, it is sufficent to consider only odd values of $q$. The following convention is used. Extending inequality (1) by $u \in\{0,1\}$, one gets $P_{\left(K, d^{K}\right)}(u)=0$ and hence $P_{q}^{L}(0)=P_{q}^{L}(1)=0$.

Theorem 6 Let $q, u \in \mathbf{N} \backslash\{1\}$ and $q$ be odd. Let $u=a q+b$ with $a, b \in \mathbf{N}_{0}$ and $b<q$. Then

$$
P_{q}^{L}(u) \geq a(u+b) \frac{q^{2}-1}{8}+P_{q}^{L}(b)
$$

Proof: Put $J_{s}:=\{0, \ldots, q-1\} \times\{s\}$ with $s \in\{0, \ldots, a-1\}$ as well as $J_{a}:=\left\{\left(\left\lfloor\frac{j q}{b}\right\rfloor, a\right) \mid j \in\right.$ $\{0, \ldots, b-1\}\}$. Put $v^{(r, s)}:=r$ for all $(r, s) \in J:=\bigcup_{s=0}^{a} J_{s}$. Using the proof of Theorem 5, it follows that

$$
\sum_{\{j, k\} \subseteq \bigcup_{s=0}^{a-1} J_{s}} d_{L}\left(v^{(j)}, v^{(k)}\right)=a^{2} \sum_{\{j, k\} \subseteq J_{0}} d_{L}\left(v^{(j)}, v^{(k)}\right)=a^{2} P_{q}^{L}(q)
$$

and

$$
\sum_{\{j, k\} \subseteq J_{a}} d_{L}\left(v^{(j)}, v^{(k)}\right)=P_{q}^{L}(b)
$$


as well as

$$
\sum_{j \in \bigcup_{s=0}^{a-1} J_{s} ; k \in J_{a}} d_{L}\left(v^{(j)}, v^{(k)}\right)=2 a b \sum_{i=0}^{\frac{q-1}{2}} i=a b \frac{q^{2}-1}{4} .
$$

Hence,

$$
P_{q}^{L}(u) \geq \sum_{\{j, k\} \subseteq J} d_{L}\left(v^{(j)}, v^{(k)}\right)=a(u+b) \frac{q^{2}-1}{8}+P_{q}^{L}(b)
$$

is valid.

One might conjecture equality in (10). The combination of the formulas (7) and (10) proves e.g. $P_{3}^{L}(5)=\left\lfloor Q_{3}^{L}(5)\right\rfloor=8<9=N_{3}^{L}(5)$ and $P_{3}^{L}(8)=\left\lfloor Q_{3}^{L}(8)\right\rfloor=21<22=N_{3}^{L}(8)$.

For some applications, let $u(d) \geq u \in \mathbf{N} \backslash\{1\}$.

(i) Let $u=3$. Inequality (2) and Theorem 2 imply the condition $3 d \leq q n$. Theorem 5 shows that inequality (1) cannot improve this condition.

(ii) Let $u=4$ and use (2). If $q$ is even then $3 d \leq q n$ follows again. If $q$ is odd then the stronger condition $6 d \leq(2 q-1) n$ follows. In both cases, an improvement by $(1)$ is impossible.

(iii) Let $u=5$. Inequality (2) implies $10 d \leq 3 q n$. Only in case of $q=3$, an improvement by (1) is possible: $5 d \leq 4 n$.

(iv) Let $q$ be even and $u$ be odd. Then inequality (1) implies the same condition for $u$ and $u+1$, since $\left(\begin{array}{l}u \\ 2\end{array}\right)^{-1} P_{q}^{L}(u)=\left(\begin{array}{c}u+1 \\ 2\end{array}\right)^{-1} P_{q}^{L}(u+1)$.

(v) Let $q$ be even. Then $\left(\begin{array}{l}u \\ 2\end{array}\right)^{-1} P_{q}^{L}(u)>\frac{q}{4}$ and $\lim _{u \rightarrow \infty}\left(\begin{array}{l}u \\ 2\end{array}\right)^{-1} P_{q}^{L}(u)=\frac{q}{4}$. Hence, inequality (1) turns out to be a tautology iff $4 d \leq q n$.

\section{References}

[1] Berlekamp, E.R.: Algebraic Coding Theory, McGraw-Hill, New York, 1968.

[2] Bogdanova, G.T. / Brouwer, A.E. / Kapralov, S.N. / Östergård, P.R.J.: ErrorCorrecting Codes over an Alphabet of Four Elements, Des. Codes Cryptogr., 23 (2001), 333-342.

[3] Lee, C.Y.: Some Properties of Nonbinary Error-Correcting Codes, IRE Trans. Inform. Theory, 4 (1958), 77-82.

[4] Mackenzie, C. / Seberry, J.: Maximal Ternary Codes and Plotkin's Bound, Ars Comb., 17A (1984), 251-270.

[5] MacWilliams, F.J. / Sloane, N.J.A.: The Theory of Error-Correcting Codes, NorthHolland, Amsterdam, New York, Oxford, 1977. 
[6] Plotkin, M.: Binary Codes with Specified Minimum Distance, Univ. Penn. Res. Div. Report 51-20 (1951); IRE Trans. Inform. Theory, 6 (1960), 445-450.

[7] Quistorff, J.: Simultane Untersuchung mehrfach scharf transitiver Permutationsmengen und MDS-Codes unter Einbeziehung ihrer Substitute, Habilitationsschrift, Univ. Hamburg, 1999; Shaker Verlag, Aachen, 2000.

[8] Răduică, M.: Marginile Plotkin si Ioshi relativ la coduri arbitrar metrizate, Bul. Univ. Braşov, C 22 (1980), 115-120.

[9] Vaessens, R.J.M. / Aarts, E.H.L. / van Lint, J.H.: Genetic Algorithms in Coding Theory - a Table for $A_{3}(n, d)$, Discrete Appl. Math., 45 (1993), 71-87.

[10] Wyner, A.D. / Graham, R.L.: An Upper Bound on Minimum Distance for a $k$-ary Code, Inform. Control, 13 (1968), 46-52. 